\title{
Ambulant vor stationär
}

\section{Sozialpsychiatrische Krisenprävention und Krisenhilfe - Beispiel Bochum}

\author{
Jörg Kalthoff
}

The principle outpatient care preferred to in-patient care is applicable as well on the prevention of crisis as the intervention in case of occurring crisis. The socio-psychiatric service in Bochum is a good example in this regard.

Le principe du traitement par consultation plutôt que l'hospitalisation s'applique aussi pour la prévention des situations de crise et pour l'intervention au cas où surgit une crise. Le service socio psychiatrique de Bochum est un bel exemple à cet égard.

Dr. Jörg Kalthoff ist Leiter des Sozialpsychiatrischen Dienstes der Stadt Bochum.

E-Mail JKalthoff@bochum.de
Die Leitlinie »ambulant vor stationär « ist auch in der Krisenprävention und Krisenintervention durchführbar, wie das Beispiel des erfolgreichen Bochumer Sozialpsychiatrischen Dienstes zeigt.

Vor 25 Jahren begann alles mit einer »Krise« der Psychiatrischen Versorgung in Bochum. Gemeindepsychiatrische Einrichtungen waren nicht vorhanden, ein Sozialpsychiatrischen Dienst $(\mathrm{SpD})$ existierte noch nicht. Stattdessen dominierten im Gesundheitsamt Kontroll- und manchmal Zwangsmaßnahmen. Diese Krise eröffnete jedoch die Chance zu einem radikalen Neuaufbau. In gemeinsamer Abstimmung von betroffenen Menschen und Fachleuten, Politik und Verwaltung, öffentlichem Gesundheitsdienst und freien Trägern wurden in Bochum ambulante Hilfen entwickelt, die insbesondere die Krisenvermeidung in den Fokus rückten.

Von den inzwischen sechs Teams des Sozialpsychiatrischen Dienstes Bochum (drei Allgemeinpsychiatrie, drei Sucht) sind drei bei der Stadt Bochum und drei bei unterschiedlichen freien Trägern angesiedelt. Durch regelmäßige gemeinsame Koordinierung und die personelle Klammer des Verfassers als fachlicher Leiter aller Teams - auch der bei den freien Trägern - ist sichergestellt, dass jeweils nach den gleichen Standards und Leitlinien gearbeitet wird.

\section{Niedrigschwelligkeit}

Statt eines zentralen Dienstes für ganz Bochum (knapp 400.000 Einwohner) findet sich von Anfang an die räumliche Sektorisierung des Sozialpsychiatrischen Dienst mit drei Sektoren (West, Mitte, Ost). Die Beratungsstellen sind also dezentral angesiedelt, die Ratsuchenden haben kurze Wege. Auf der anderen Seite sind die Teams des Sozialpsychiatrischen Dienst in ihren Stadtbezirken präsent, kennen die psychiatrischen und sonstigen Hilfsangebote und arbeiten eng mit ihnen zusammen.

Zur räumlichen Niedrigschwelligkeit tritt die inhaltliche : Die Beratungsstellen haben überwiegend nach dem Prinzip der offenen Tür einen großen Aufenthaltsund Gruppenraum, der für Besucher ohne Voranmeldung nutzbar ist. Hier halten sich Menschen mit psychischer Erkrankung auf, um der Einsamkeit zu entfliehen, um sich mit anderen Menschen zu treffen, Kaffee zu trinken, Gesellschaftsspiele zu machen, sich zu unterhalten.

Als »Eintrittskarte « müssen keine therapeutischen Gespräche geführt werden, diese ergeben sich eher nach einer Annäherung über Kontakte zwischen Tür und Angel. Über Mund-zu-Mund-Propaganda kommt es zur Werbung bei anderen Betroffenen und zur Annäherung an das Hilfesystem ohne hohe Schwellen. Die hohe Selbstmeldequote und sehr niedrige Abbruchquote führen wir auch auf diese Niedrigschwelligkeit der Angebote zurück.

\section{Stärkung der Ressourcen}

In akuten Krisensituationen steht oft zunächst die Sicherung der Lebensgrundlagen im Vordergrund: gesundheitliche und finanzielle Grundlagen, Sicherung der Wohnung. Stellt sich danach heraus, dass weitere Schritte zur Vermeidung erneuter Krisen erforderlich sind, werden entsprechende Hilfen gemeinsam mit den betroffenen Menschen geplant und es wird bei den gesunden Anteilen angesetzt (»verhandeln statt behandeln «).

Neben den Einzelgesprächen haben hier die Gruppen, die in allen Teams angeboten werden, eine wichtige Funktion. Hier kommt es zum sozialen Lernen, die oft jahrelange Isolation wird aufgebrochen, Kontakte werden geknüpft und intensiviert. Auch die Tatsache, anderen 
Betroffenen selbst weiterhelfen zu können und wieder "geben « zu können, trägt sehr zur Stärkung des Selbstbewusstseins bei.

Als wesentliche Bereicherung und Besonderheit in Bochum sind in allen Beratungsstellen ambulante ergotherapeutische Angebote aufgebaut worden. Über das gemeinsame Tun kommt es nochmals auf einer anderen Ebene zu sozialen Kontakten als bei rein verbalen Angeboten. Die Tage und Wochen werden neu strukturiert, für viele kommt es hier zur neuen Sinn- und Hobbyfindung. Neben gemeinsamen Projektgruppen werden Dinge für den Eigenbedarf und Auftraggeber herge- sonders komplexer psychiatrischer und sozialer Problematik und chronischem Verlauf. Daher dominieren bei den Diagnosen entsprechend der Pflichtversorgung die schizophrenen Psychosen und die chronisch mehrfach geschädigten $\mathrm{Ab}$ hängigkeitserkrankten mit jeweils einem Drittel. Der Krankheitsverlauf beträgt im Mittel zehn bis 20 Jahre. Entsprechend verteilen sich auch die Betreuungszeiten neben rund 30 Prozent Erstkontakten relativ gleichmäßig bis zu zehn Jahren. Überdurchschnittlich häufig kam es unter den Betreuten in der Vergangenheit zu mehreren stationären Aufenthalten, oft auch unter Zwangsbedingungen.

\section{»Die Krisenprävention muss Vorrang haben vor der Krisenintervention"}

stellt. Wertschätzung für die eigene Leistung wird oft nach Jahren erstmals wieder erzielt. Dieses ist häufig auch der Motor, weitere Schritte hin zur beruflichen Wiedereingliederung zu tun. Über dieses Angebot mit stufenweiser Steigerung der Anforderungen gelingt es Jahr für Jahr, einen Teil der Besucher wieder in den Arbeits- und Rehaprozess einzugliedern. Zusätzlich hat sich diese äußere Struktur in erneuten Krisenzeiten als sehr hilfreich für die innere Struktur entwickelt, so dass teure Krankenhausaufenthalte vermieden werden konnten.

\section{Priorität für die Schwächsten}

All diese Angebote sind ein Lernfeld für viele um zu üben, wieder auf Menschen zuzugehen und Ängste zu überwinden. Im zweiten Schritt folgt zunächst unter Begleitung der Fachleute und anderer Betroffener die Teilhabe über den Psychiatriebereich hinaus (z. B. Volkshochschule, Kirchengemeinde, Sport, kulturelle Angebote). Im dritten Schritt ist dann häufig wieder so viel Sicherheit gewonnen, dass diese Angebote allein genutzt werden können. Sehr wichtig für die Wiedereingliederung ist eine jährlich stattfindende zehntägige Rehabilitationsmaßnahme im Selbstversorger-Haus mit intensiveren Trainingsmöglichkeiten.

Im Zentrum unserer Bemühungen stehen die Bürgerinnen und Bürger mit be-
Nach unserer Erfahrung benötigen chronische Klienten auch "chronische» Mitarbeiter. Gerade für sie ist es wichtig nicht zwischen einzelnen Mitarbeitern oder Berufsgruppen hin und her geschoben zu werden. Entscheidend ist eine klare persönliche Beziehung. Daher arbeitet der Sozialpsychiatrische Dienst Bochum nach dem Bezugspersonensystem, bei dem jeder Klient langfristig einem Mitarbeiter zugeordnet wird. Dieser begleitet die Betroffenen gegebenenfalls zu anderen Berufsgruppen oder weiteren Institutionen. Alle Wege, die der Klient geht, führen letztlich zur Bezugsperson zurück.

Flexibel wird die Betreuungsintensität dem Bedarf angepasst und schwankt zwischen sehr häufigen Kontakten in Krisenzeiten und langgestreckten Kontakten in besseren Zeiten. Wichtig ist jedoch, dass der Kontakt nicht abreißt und im Krisenfall sehr schnell wieder intensiviert und genutzt werden kann.

Gerade für die Schwächsten ist ein ganzheitliches Angebot mit unterschiedlicher Nutzungsmöglichkeit wichtig. Sie sind in der Regel davon überfordert, an unterschiedlichen Stellen für sich selbst Gesundheitsleistungen zusammenzustellen und in Anspruch zu nehmen. Daher verstecken sich beim Sozialpsychiatrischen Dienst hinter derselben Eingangstür verschiedene Leistungen wie Einzelberatung, Angehörigenberatung, medikamentöse Behandlung, ambulante Ergotherapie, Kontaktfindung usw. Je nach Notwendigkeit und Situation können diese Angebote unterschiedlich in Anspruch genommen werden. Auch die Möglichkeit innerhalb dieser Angebote am gleichen Ort wechseln zu können und trotzdem die gleiche Bezugsperson zu behalten, hat sehr zur kontinuierlichen Betreuung vieler Menschen beigetragen. Ohne diese intensive Nutzung des Sozialpsychiatrischen Dienst wären viele Besucher in den Jahren dauerhaft Wohnheimbewohner geworden.

\section{Alternativen zur Hospitalisierung}

Zur Umsetzung der Leitlinie "ambulant vor stationär « ist es wichtig, die Hilfen, die ansonsten stationär angeboten würden, durch den Sozialpsychiatrischen Dienst auch ambulant bereitstellen zu können. So komplex wie die Problemlage gerade der Schwächsten ist, so komplex und berufsgruppenübergreifend müssen die ambulanten Hilfsangebote sein. Im multiprofessionellen Team treten in Bochum zu den klassischen Berufsgruppen eines Sozialpsychiatrischen Dienstes (Medizin, Sozialarbeit) auch Krankenpflegepersonal, Ergotherapeuten und Psychologen. Zum einen kann so der spezifische Hilfebedarf der Betroffenen noch exakter erfüllt werden, zum anderen kommt es im Team zur wechselseitigen Kompetenzerweiterung der einzelnen Berufsgruppen.

Viele Patienten sind nicht in der Lage oder bereit, sich im Wartezimmer, beispielsweise eines niedergelassenen Nervenarztes, aufzuhalten. Sie zeigen ein eingeschränktes Hilfesuchverhalten. Die Folgen davon waren häufig erneute Erkrankungen und eine stationäre Behandlungsbedürftigkeit. Durch eine Vereinbarung mit den gesetzlichen Krankenkassen hat der Bochumer Sozialpsychiatrischen Dienst seit 1992 die Möglichkeit, zur Krisenintervention gerade dieses Personenkreises auch medikamentös zu behandeln. Vor Ort konnte in einer akuten Notsituation in der Regel im Hausbesuch eine Beziehung aufgebaut werden und direkt auch behandelt werden. Damit wurden Krankenhausbehandlungen vermieden. Auch bei typischen »Drehtürpatienten ", die immer wieder zuvor nach Krankenhausbehandlung die Medikation absetzten, kurzfristig wieder dekompensierten und wieder untergebracht werden 
mussten, bestand diese Behandlungsmöglichkeit des Sozialpsychiatrischen Dienstes.

Darüber entstanden zum Teil über zehnjährige krankenhausfreie Zeiten, nachdem es zuvor regelmäßig wenigstens einmal pro Jahr zu stationären Aufenthalten gekommen war. Auch hier hat sich die Niedrigschwelligkeit als wichtig herausgestellt, so dass beispielsweise die größte Depotsprechstunde zeitlich mit dem größten Kontaktklubangebot verknüpft wurde. Dieses erfolgreiche Modell, das für viele Klienten den entscheidenden Schritt zur Wiedereingliederung ins normale Leben dargestellt hat, kann leider mit einigen Krankenkassen aus formalen Gründen nicht mehr fortgesetzt werden.

\section{Krisenvermeidung}

Die Krisenprävention hat Vorrang vor der Krisenintervention. Daher bemühen wir uns besonders um diejenigen Menschen, bei denen in der Vergangenheit häufig Krisen aufgetreten sind. Durch die beschriebenen Arbeitsweisen sind die Krisen bei diesem Personenkreis erheblich zurückgegangen. Inzwischen wurde für die meisten ein so gutes Netz mit Fachleuten und anderen Betroffenen geknüpft, dass mögliche Krisen jeweils im Vorfeld abgefangen werden können.

Treten trotz der beschriebenen Hilfsmöglichkeiten dennoch Krisen auf, ist die personelle Vertrautheit zu den Mitarbeiterinnen und Mitarbeitern besonders wichtig. Hier wird auf gemeinsame positive Erfahrungen zurückgegriffen. Bei guter gegenseitiger Kenntnis können häufig in Krisensituationen Lösungen gefunden werden, oft flexibel und unbürokratisch, manchmal über die ursprünglich festgelegten Aufgabenbereiche hinaus.

Die besondere Möglichkeit des Sozialpsychiatrischen Dienstes, auch ohne besonderen Auftrag tätig zu werden und Hausbesuche durchzuführen, bietet gute Möglichkeiten. Gerade psychotisch erkrankte Betroffene sind in Krisen gegenüber dem Angebot von Hilfe oft sehr ambivalent. Durch die Geh-Struktur wird die Annahme erheblich erleichtert. Dabei ist die Arbeitsweise nicht nur aufsuchend, sondern auch nachgehend: Nach möglicherweise zunächst missglückten Kontaktversuchen wird nicht aufgegeben, sondern es werden andere Kontaktwege gesucht. Hierfür gibt es nach Bewältigung der Krisen in der Regel sehr positive Rückmeldungen der betroffenen Menschen.

\section{Krisenintervention}

Mit den beschriebenen Strukturen können auch in Bochum nicht alle Krisen im Vorfeld vermieden werden. Insbesondere bei uns noch nicht bekannten Menschen mit psychischen Erkrankungen kommt es häufig zu Fremdmeldungen aus dem sozialen Umfeld oder durch die Polizei. Für diese Krisen, oft mit Eigen- oder Fremdgefährdung, halten alle Teams des Sozialpsychiatrischen Dienstes jeweils einen Bereitschaftsdienst vor, der umgehende telefonische oder persönliche Beratung anbietet. Im Bedarfsfall kommt er auch zur Krisenintervention sofort ins Haus. Dies geschieht in der Regel unter Beteiligung der ärztlichen Kolleginnen und Kollegen. Auch hier gelingt es - vor Ort - in der weit überwiegenden Zahl ambulante Lösungsmöglichkeiten zu entwickeln und Zwangsunterbringungen zu vermeiden. Die Quote der Verhinderung von Unterbringungen im Bochumer Sozialpsychiatrischen Dienst liegt bei 92 Prozent. Die deutliche Priorität der Hilfen zeigt sich daran, dass über 45.000 Betreuungskontakten im Jahr weniger als 20 Zwangseinweisungen nach dem nordrhein-westfälischen Gesetz über Hilfen und Schutzmaßnahmen bei psychischen Krankheiten (PsychKG) gegenüberstehen.

Das Bündel aus Krisenprävention und Krisenintervention in Kooperation mit den anderen gemeindepsychiatrischen Trägern hat dazu geführt, dass die PsychKG-Einweisungsrate in Bochum (41 auf 100.000 Einwohner) deutlich unter dem Durchschnitt anderer Großstädte liegt: Sie beträgt weit weniger als die Hälfte der durchschnittlichen Einweisungsrate in Nordrhein-Westfalen.

\section{Fazit}

In den letzten 25 Jahren hat sich in Bochum aus dem Nichts heraus ein beispielhafter Sozialpsychiatrischer Dienst entwickelt. Priorität haben dabei Klienten mit besonders komplexer Problematik und chronischem Verlauf. Die Krisenver- meidung hat Vorrang vor der Krisenintervention. Für viele Betroffenen sind diese Angebote Alternativen zur Langzeithospitalisierung oder zur Drehtürpsychiatrie. Auch Betroffene mit eingeschränktem Hilfesuchverhalten können durch Niedrigschwelligkeit der Angebote zur Nutzung motiviert werden.

In den Jahren wurden rund 60.000 Bochumer und ihr soziales Umfeld entsprechend beraten, bei fast 30.000 Betroffenen kam es zu intensiverer Begleitung, Betreuung und manchmal Behandlung. Die Zahl langfristiger stationärer Unterbringungen ist auf ein Fünftel reduziert worden, die Zwangseinweisungsrate für eine Großstadt ausgesprochen niedrig.

Diese Resultate zeigen: »Ambulant vor stationär « ist auch in der sozialpsychiatrischen Krisenprävention und Krisenhilfe bei Menschen mit komplexen psychiatrischen Problemen möglich.

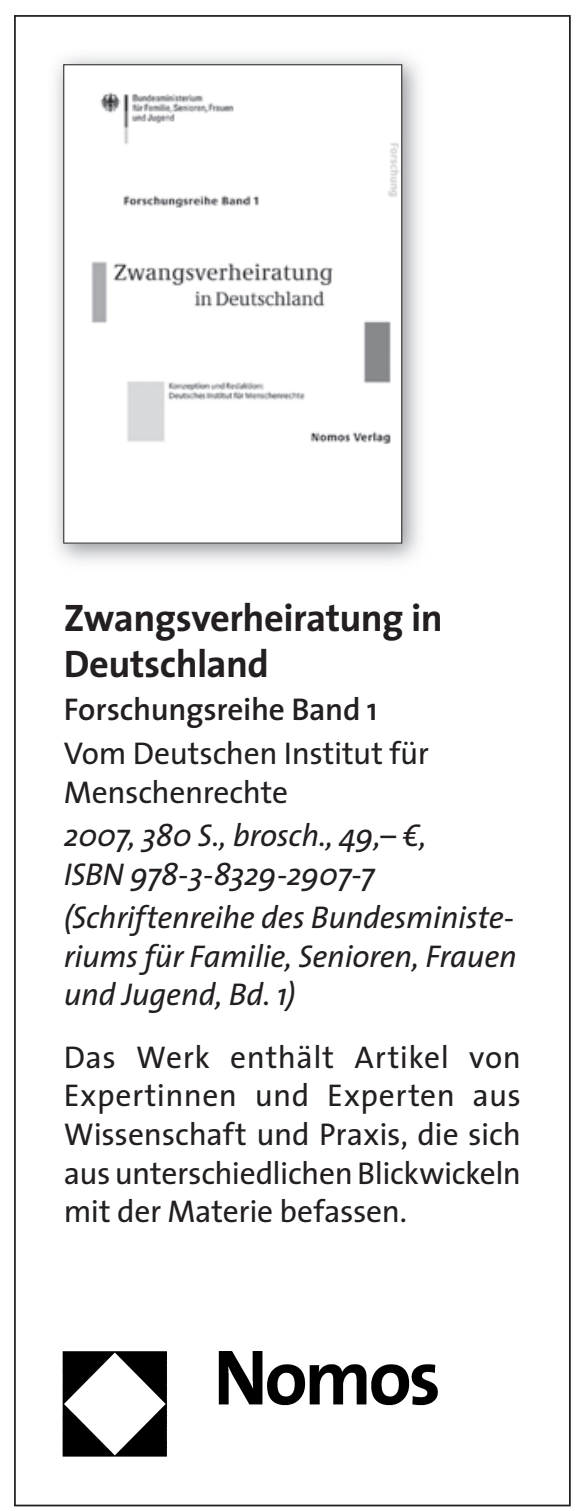

